\title{
Some Symmetric Curvature Conditions on Kenmotsu Manifolds
}

\author{
Riddhi Jung Shah \\ Department of Mathematics, Janata Campus, Nepal Sanskrit University, Nepal \\ Correspondence to: Riddhi Jung Shah, email: shahrjgeo@gmail.com
}

\begin{abstract}
In this paper, we study locally and globally $\varphi$-symmetric Kenmotsu manifolds. In both curvature conditions, it is proved that the manifold is of constant negative curvature - 1 and globally $\varphi$-Weyl projectively symmetric Kenmotsu manifold is an Einstein manifold. Finally, we give an example of 3-dimensional Kenmotsu manifold.
\end{abstract}

Keywords: Kenmotsu manifold, Locally $\varphi$-symmetric, Globally $\varphi$-symmetric, Weyl projective curvature tensor

\section{Introduction}

Tanno [8] classified connected almost contact metric manifolds whose automorphism groups have the maximum dimension. For such a manifold $M$, the sectional curvature of plane sections containing $\xi$ is a constant, say $c$, where $\xi$ is a global vector field (or contravariant vector field or Reeb vector field). If $c>0, M$ is a homogeneous Sasakian manifold of constant $\varphi$-sectional curvature. If $c=0, M$ is the product of a line or circle with a Kaehler manifold of constant holomorphic curvature. If $c<0, M$ is a warped product space $R \times_{f} C^{n}$. In [5], Kenmotsu abstracted the differential geometric properties of the case if $c<0$ and also introduced the notion of a class of almost contact Riemannian manifolds with some special conditions. We call this type of manifold, a Kenmotsu manifold.

Takahashi [7] introduced the notion of locally $\varphi$-symmetric Sasakian manifold as a weaker version of local symmetry of such manifold. In this paper, we study locally $\varphi$-symmetric Kenmotsu manifold, globally $\varphi$-symmetric Kenmotsu manifold and globally $\varphi$-Weyl projectively symmetric Kenmotsu manifold. In first two cases, we have obtained the result that the manifold is of constant negative curvature - 1 . In next condition, it is shown that the manifold is an Einstein manifold with scalar curvature $r=n(n-1)$.

\section{Preliminaries}

Let $M$ be an $n$-dimensional (where $n=2 m+1$ ) almost contact manifold with an almost contact metric structure $(\varphi, \xi, \eta, g)$, where $\varphi$ is a $(1,1)$ tensor field, $\xi$ is a Reeb vector field (or contravariant vector field), $\eta$ is a 1 -form and $g$ is a compatible Riemannian metric such that

$$
\begin{gathered}
\varphi^{2}(X)=-X+\eta(X) \xi, \eta(\xi)=1, \varphi \xi=0, \eta(\varphi X)=0 \\
g(\varphi X, \varphi Y)=g(X, Y)-\eta(X) \eta(Y) \\
g(X, \xi)=\eta(X)
\end{gathered}
$$

for all $X, Y \in T(M)$ 1, 2. An almost contact metric manifold $\left(M^{n}, g\right)$ is said to be a Kenmotsu manifold if the conditions

$$
\begin{gathered}
\left(\nabla_{X} \varphi\right) Y=g(\varphi X, Y) \xi-\eta(Y) \varphi X \\
\nabla_{X} \xi=X-\eta(X) \xi
\end{gathered}
$$

hold in $M$, where $\nabla$ is the Levi-Civita connection of $g[5]$.

In an $n$-dimensional $(n=2 m+1)$ Kenmotsu manifold, the following relations hold [5]

$$
\left(\nabla_{X} \eta\right) Y=g(X, Y)-\eta(X) \eta(Y)
$$




$$
\begin{gathered}
R(X, Y) \xi=\eta(X) Y-\eta(Y) X, \\
S(X, \xi)=-(n-1) \eta(X), \\
S(\varphi X, \varphi Y)=S(X, Y)+(n-1) \eta(X) \eta(Y)
\end{gathered}
$$

for any vector fields $X, Y$ on $M$, where $R$ and $S$ are the Riemannian curvature tensor and the Ricci tensor respectively.

Definition 2.1. A Kenmotsu manifold $\left(M^{n}, g\right)$ is said to be a locally $\varphi$-symmetric manifold if the condition

$$
\varphi^{2}\left(\left(\nabla_{W} R\right)(X, Y) Z\right)=0
$$

holds for any vector fields $X, Y, Z, W$ orthogonal to $\xi$, that is for any horizontal vector fields $X, Y, Z, W$.

This notion was introduced by Takahashi [7] for Sasakian manifold.

Definition 2.2. An $n$-dimensional Kenmotsu manifold $M$ is said to be globally $\varphi$-symmetric if it satisfies the condition

$$
\varphi^{2}\left(\left(\nabla_{W} R\right)(X, Y) Z\right)=0
$$

for arbitrary vector fields $X, Y, Z$ and $W$ on $M$. [3]

The Weyl projective curvature tensor $P$ of type $(1,3)$ on a Riemannian manifold $\left(M^{n}, g\right)$ is defined by

$$
P(X, Y) Z=R(X, Y) Z-\frac{1}{n-1}[S(Y, Z) X-S(X, Z) Y]
$$

for any $X, Y, Z \in \chi(M)$, the set of vector fields.

Definition 2.3. A Kenmotsu manifold $M$ of dimension $n$ is said to be globally $\varphi$-Weyl projectively symmetric if the Weyl projective curvature tensor $P$ satisfies

$$
\varphi^{2}\left(\left(\nabla_{W} P\right)(X, Y) Z\right)=0
$$

for all vector fields $X, Y, Z, W \in \chi(M)$.

\section{$3 \quad$ Results and Discussions}

Theorem 3.1. A Kenmotsu manifold $\left(M^{n}, g\right)$ is locally $\varphi$-symmetric if and only if

$$
\left(\nabla_{W} R\right)(X, Y) Z=g(R(X, Y) Z, W) \xi
$$

for any horizontal vector fields $X, Y, Z$ and $W$.

Proof. Let us consider an $n$-dimensional Kenmotsu manifold which satisfies the condition 2.10). Then by the use of 2.1], the relation 2.10 yields

$$
\left(\nabla_{W} R\right)(X, Y) Z+g\left(\left(\nabla_{W} R\right)(X, Y) \xi, Z\right) \xi=0 .
$$

From 2.7), we have

$$
\left(\nabla_{W} R\right)(X, Y) \xi=\left(\nabla_{W} \eta\right)(X) Y+\eta(X) \nabla_{W} Y-\left(\nabla_{W} \eta\right)(Y) X-\eta(Y) \nabla_{W} X .
$$

In view of 2.6 and 3.2 , we obtain

$$
\begin{aligned}
\left(\nabla_{W} R\right)(X, Y) \xi= & g(X, W) Y-g(Y, W) X-\eta(X) \eta(W) Y \\
& +\eta(X) \nabla_{W} Y+\eta(Y) \eta(W) X-\eta(Y) \nabla_{W} X
\end{aligned}
$$


For horizontal vectors $X, Y, W$, the relation 3.3 reduces to

$$
\left(\nabla_{W} R\right)(X, Y) \xi=g(X, W) Y-g(Y, W) X .
$$

Using (3.4) in the relation 3.1, we get

$$
\left.\left(\nabla_{W} R\right)(X, Y) Z\right)+g(g(X, W) Y-g(Y, W) X, Z) \xi=0
$$

or,

$$
\left(\nabla_{W} R\right)(X, Y) Z-g(R(X, Y) Z, W) \xi=0
$$

this implies

$$
\left(\nabla_{W} R\right)(X, Y) Z=g(R(X, Y) Z, W) \xi
$$

for any horizontal vector fields $X, Y, Z$ and $W$. Next, if the relation 3.5 holds for any vector fields $X, Y, Z, W$ orthogonal to $\xi$, it follows from $\varphi \xi=0$ that 2.10 holds and hence the manifold is locally $\varphi$-symmetric. This completes the proof of the theorem.

From 3.5 , it also follows that if $\left(\nabla_{W} R\right)(X, Y) Z=0$, then $R(X, Y) Z=0$ since $W$ and $\xi$ are non-zero. Thus, we have a corollary

Corollary 3.2. If an n-dimensional Kenmotsu manifold is locally symmetric, then the manifold is flat.

Again, in corollary 6 of proposition 5 Kenmotsu [5] proved that if a Kenmotsu manifold is locally symmetric, then it is of constant negative curvature - 1 .

Theorem 3.3. Let $M$ be an $n$-dimensional Kenmotsu manifold. If $M$ is globally $\varphi$-symmetric, then it is locally symmetric.

Proof. Let $M$ be an $n$-dimensional Kenmotsu manifold. Suppose that the condition (2.11) holds. Then from (2.1) and (2.11) we obtain

$$
-\left(\nabla_{W} R\right)(X, Y) Z+\eta\left(\left(\nabla_{R} R\right)(X, Y) Z\right) \xi=0
$$

or,

$$
\left(\nabla_{W} R\right)(X, Y) Z+g\left(\left(\nabla_{W} R\right)(X, Y) \xi, Z\right) \xi=0
$$

By the use of equation (3.3) of proposition 5 of [5] as

$$
\left(\nabla_{Z} R\right)(X, Y) \xi=g(Z, X) Y-g(Z, Y) X-R(X, Y) Z
$$

in the relation 3.7 , we get

$$
\left.\left(\nabla_{W} R\right)(X, Y) Z+g(X, W) g(Y, Z) \xi-g(Y, W) g(X, Z) \xi-g(R(X, Y) W), Z\right) \xi=0 .
$$

In view of 2.7), relation 3.8 reduces to

$$
\left(\nabla_{W} R\right)(X, Y) Z=0
$$

Hence the theorem is proved.

From Theorem 3.3 and corollary 6 of [5], we can state next theorem

Theorem 3.4. If an $n$-dimensional Kenmotsu manifold $M$ is globally $\varphi$-symmetric, then it is of constant negative curvature - 1 .

Theorem 3.5. Let $\left(M^{n}, g\right)$ be a Kenmotsu manifold. If $M$ is globally $\varphi$-Weyl projectively symmetric, then it is an Einstein manifold with scalar curvature $r=n(n-1)$. 
Proof. Let us consider $M$ is a globally $\varphi$-Weyl projectively symmetric manifold. Then 2.13 holds. Now, using 2.1, we obtain

$$
-\left(\nabla_{W} P\right)(X, Y) Z+\eta\left(\left(\nabla_{W} P\right)(X, Y) Z\right) \xi=0
$$

Differentiating 2.12 covariantly with respect to $W$, we get

$$
\left.\left(\nabla_{W} P\right)(X, Y) Z=\left(\nabla_{W} R\right)(X, Y) Z-\frac{1}{n-1}\left[\left(\nabla_{W} S\right)(Y, Z) X-\left(\nabla_{W} S\right)(X, Z) Y\right)\right] .
$$

In view of 3.10 and 3.11 , we get

$$
\begin{aligned}
0= & -g\left(\left(\nabla_{W} R\right)(X, Y) Z, U\right)+\frac{1}{n-1}\left[\left(\nabla_{W} S\right)(Y, Z) g(X, U)-\left(\nabla_{W} S\right)(X, Z) g(Y, U)\right] \\
& +\eta\left(\left(\nabla_{W} R\right)(X, Y) Z\right) \eta(U)-\frac{1}{n-1}\left[\left(\nabla_{W} S\right)(Y, Z) \eta(X)-\left(\nabla_{W} S\right)(X, Z) \eta(Y)\right] \eta(U) .
\end{aligned}
$$

Let $\left\{e_{i}\right\}, i=1,2, \ldots, n$ be an orthonormal basis of the tangent space at any point of the manifold. Putting $X=U=e_{i}$, in 3.12 and summing over $i, 1 \leq i \leq n$, we get

$$
\begin{aligned}
0= & -\left(\nabla_{W} S\right)(Y, Z)+\frac{1}{n-1}\left[n\left(\nabla_{W} S\right)(Y, Z)-\left(\nabla_{W} S\right)(Y, Z)\right]+\eta\left(\left(\nabla_{W} R\right)\left(e_{i}, Y\right) Z\right) \eta\left(e_{i}\right) \\
& -\frac{1}{n-1}\left[\left(\nabla_{W} S\right)(Y, Z)-\left(\nabla_{W} S\right)(Z, \xi) \eta(Y)\right]
\end{aligned}
$$

or,

$$
0=\eta\left(\left(\nabla_{W} R\right)\left(e_{i}, Y\right) Z\right) \eta\left(e_{i}\right)-\frac{1}{n-1}\left[\left(\nabla_{W} S\right)(Y, Z)-\left(\nabla_{W} S\right)(Z, \xi) \eta(Y)\right]
$$

Putting $Z=\xi$ in 3.13 , we obtain

$$
0=\eta\left(\left(\nabla_{W} R\right)\left(e_{i}, Y\right) \xi\right) \eta\left(e_{i}\right)-\frac{1}{n-1}\left(\nabla_{W} S\right)(Y, \xi)+\frac{1}{n-1}\left(\nabla_{W} S\right)(\xi, \xi) \eta(Y)
$$

Now, we have

$$
\eta\left(\left(\nabla_{W} R\right)\left(e_{i}, Y\right) \xi\right) \eta\left(e_{i}\right)=g\left(\left(\nabla_{W} R\right)\left(e_{i}, Y\right) \xi, \xi\right) g\left(e_{i}, \xi\right)
$$

Again, we get

$$
\begin{aligned}
\left.g\left(\nabla_{W} R\right)\left(e_{i}, Y\right) \xi, \xi\right)= & g\left(\nabla_{W} R\left(e_{i}, Y\right) \xi, \xi\right)-g\left(R\left(\nabla_{W} e_{i}, Y\right) \xi, \xi\right) \\
& -g\left(R\left(e_{i}, \nabla_{W} Y\right) \xi, \xi\right)-g\left(R\left(e_{i}, Y\right) \nabla_{W} \xi, \xi\right)
\end{aligned}
$$

Since $\left\{e_{i}\right\}$ is an orthonormal basis $\nabla_{W} e_{i}=0$. From 2.7 we have

$$
\begin{aligned}
g\left(R\left(e_{i}, \nabla_{W} Y\right) \xi, \xi\right) & =g\left(\eta\left(e_{i}\right) \nabla_{W} Y-\eta\left(\nabla_{W} Y\right) e_{i}, \xi\right) \\
& =\eta\left(e_{i}\right) \eta\left(\nabla_{W} Y\right)-\eta\left(\nabla_{W} Y\right) \eta\left(e_{i}\right) \\
& =0
\end{aligned}
$$

We know that if $R$ is the Riemannian curvature tensor of a Riemannian manifold $(M, g)$ 3, 4, 6], we have

$$
g(R(X, Y) Z, U)=-g(R(Z, U) Y, X)
$$

Thus, $g\left(R\left(e_{i}, Y\right) \xi, \xi\right)+g\left(R(\xi, \xi) Y, e_{i}\right)=0$ and we get

$$
g\left(\nabla_{W} R\left(e_{i}, Y\right) \xi, \xi\right)+g\left(R\left(e_{i}, Y\right) \xi, \nabla_{W} \xi\right)=0 .
$$

Using above relations in 3.15, we obtain $g\left(\left(\nabla_{W} R\right)\left(e_{i}, Y\right) \xi, \xi\right) \eta\left(e_{i}\right)=0$ and the equation 3.14 reduces to

$$
\left(\nabla_{W} S\right)(Y, \xi)-\left(\nabla_{W} S\right)(\xi, \xi) \eta(Y)=0
$$


Now, we have

$$
\begin{aligned}
\left(\nabla_{W} S\right)(Y, \xi) & =\nabla_{W} S(Y, \xi)-S\left(\nabla_{W} Y, \xi\right)-S\left(Y, \nabla_{W} \xi\right) \\
& =-(n-1) \nabla_{W} \eta(Y)+(n-1) \eta\left(\nabla_{W} Y\right)-S(Y, W)+\eta(W) S(Y, \xi) \\
& =-(n-1)\left(\nabla_{W} \eta\right)(Y)-S(Y, W)-(n-1) \eta(Y) \eta(W) \\
& =-(n-1)\{g(W, Y)-\eta(Y) \eta(W)\}-S(Y, W)-(n-1) \eta(W) \eta(Y) \\
& =-S(Y, W)-(n-1) g(W, Y) .
\end{aligned}
$$

So, putting $Y=\xi$ in above relation, we get

$$
\left(\nabla_{W} S\right)(\xi, \xi)=0
$$

Using above two relations in 3.16 , we obtain

$$
S(W, Y)=(n-1) g(W, Y) .
$$

Now, taking an orthonormal frame field at any point of the manifold and contracting over $W$ and $Y$ in 3.17 , we get

$$
r=n(n-1)
$$

where $r$ is the scalar curvature.

In view of (3.17) and (3.18), the theorem is proved.

\section{Example of 3-dimensional Kenmotsu Manifold}

Let us consider 3-dimensional manifold $M=\left\{(x, y, z) \in R^{3}\right\}, z \neq 0$ where $(x, y, z)$ are the standard coordinates of $R^{3}$. Let $\left\{E_{1}, E_{2}, E_{3}\right\}$ be a linearly independent global frame on $M$ defined by

$$
E_{1}=z \frac{\partial}{\partial x}, E_{2}=z \frac{\partial}{\partial y}, E_{3}=-z \frac{\partial}{\partial z} .
$$

Let $g$ be a Riemannian metric defined by

$$
\begin{gathered}
g\left(E_{1}, E_{2}\right)=g\left(E_{2}, E_{3}\right)=g\left(E_{1}, E_{3}\right)=0 \\
g\left(E_{1}, E_{1}\right)=g\left(E_{2}, E_{2}\right)=g\left(e_{3}, E_{3}\right)=1 .
\end{gathered}
$$

Let $\eta$ be a 1 -form defined by $\eta(U)=g\left(U, E_{3}\right)$ for any $U \in \chi(M)$, the set of vector fields. Let $\varphi$ be the $(1$, 1) tensor field defined by

$$
\varphi\left(E_{1}\right)=-E_{2}, \varphi\left(E_{2}\right)=E_{1}, \varphi\left(E_{3}\right)=0 .
$$

Then, using the linearity of $\varphi$ and $g$, we have

$$
\eta\left(E_{3}\right)=1, \varphi^{2}(U)=-U+\eta(U) E_{3}, g(\varphi U, \varphi V)=g(U, V)-\eta(U) \eta(V),
$$

for any vector fields $U, V \in \chi(M)$. Thus, for $E_{3}=\xi,(\varphi, \xi, \eta, g)$ defines an almost contact metric structure on $M$. Let $\nabla$ be the Levi-Civita connection with respect to the Riemannian metric $g$. Then, by the definition of Lie bracket, we have

$$
\begin{aligned}
{\left[E_{1}, E_{3}\right] } & =E_{1} E_{3}-E_{3} E_{1} \\
& =z \frac{\partial}{\partial x}\left(-z \frac{\partial}{\partial z}\right)-\left(-z \frac{\partial}{\partial z}\right)\left(z \frac{\partial}{\partial x}\right) \\
& =-z^{2} \frac{\partial^{2}}{\partial x \partial z}+z\left(z \frac{\partial^{2}}{\partial z \partial x}+\frac{\partial}{\partial x} \times 1\right) \\
& =z \frac{\partial}{\partial x} \\
& =E_{1} .
\end{aligned}
$$


Similarly, we obtain $\left[E_{1}, E_{2}\right]=0$ and $\left[E_{2}, E_{3}\right]=E_{2}$.

Now, we have Koszul formula

$$
\begin{aligned}
2 g\left(\nabla_{U} V, W\right) & =U g(V, W)+V g(W, U)-W g(U, V) \\
& +g([U, V], W)-g([V, W], U)+g([W, U], V) .
\end{aligned}
$$

Using above Koszul formula, we obtain

$$
\begin{aligned}
2 g\left(\nabla_{E_{1}} E_{3}, E_{1}\right) & =E_{1} g\left(E_{3}, E_{1}\right)+E_{3} g\left(E_{1}, E_{3}\right)-E_{1} g\left(E_{1}, E_{3}\right) \\
& +g\left(\left[E_{1}, E_{3}\right], E_{1}\right)-g\left(\left[E_{3}, E_{1}\right], E_{1}\right)+g\left(\left[E_{1}, E_{1}\right], E_{3}\right) \\
& =2 g\left(E_{1}, E_{1}\right) .
\end{aligned}
$$

Similarly, we can calculate

$$
2 g\left(\nabla_{E_{1}} E_{3}, E_{2}\right)=0=2 g\left(E_{1}, E_{2}\right) \quad \text { and } \quad 2 g\left(\nabla_{E_{1}} E_{3}, E_{3}\right)=0=2 g\left(E_{1}, E_{3}\right) .
$$

Thus, $g\left(\nabla_{E_{1}} E_{3}, X\right)=g\left(E_{1}, X\right)$ for all $X \in \chi(M)$.

Therefore, $\nabla_{E_{1}} E_{3}=E_{1}$.

Proceeding continuously in this way, we obtain

$$
\begin{array}{r}
\nabla_{E_{1}} E_{3}=E_{1}, \nabla_{E_{1}} E_{2}=0, \nabla_{E_{1}} E_{1}=-E_{3}, \\
\nabla_{E_{2}} E_{3}=E_{2}, \nabla_{E_{2}} E_{2}=-E_{3}, \nabla_{E_{2}} E_{1}=0, \\
\quad \text { and } \nabla_{E_{3}} E_{1}=\nabla_{E_{3}} E_{2}=\nabla_{E_{3}} E_{3}=0 .
\end{array}
$$

Now, we get

$$
\begin{aligned}
\nabla_{E_{1}} E_{3}=E_{1} & =E_{1}-g\left(E_{1}, E_{3}\right) E_{3}, \\
\nabla_{E_{2}} E_{3}=E_{2} & =E_{2}-g\left(E_{2}, E_{3}\right) E_{3}, \\
\text { and } \nabla_{E_{3}} E_{3}=0 & =E_{3}-g\left(E_{3}, E_{3}\right) E_{3} .
\end{aligned}
$$

For $E_{3}=\xi$, above results become

$$
\nabla_{X} \xi=X-g(X, \xi) \xi=X-\eta(X) \xi
$$

Thus the second condition 2.5 for Kenmotsu manifold is satisfied. Again, we have

$$
\left(\nabla_{E_{1}} \varphi\right) E_{1}=\nabla_{E_{1}} \varphi E_{1}-\varphi \nabla_{E_{1}} E_{1}=\nabla_{E_{1}}\left(-E_{2}\right)-\varphi\left(-E_{3}\right)=0
$$

and

Therefore, we get

$$
g\left(\varphi E_{1}, E_{1}\right) E_{3}-g\left(E_{1}, E_{3}\right) \varphi E_{1}=g\left(-E_{2}, E_{1}\right)=0
$$

$$
\left(\nabla_{E_{1}} \varphi\right) E_{1}=g\left(\varphi E_{1}, E_{1}\right)-g\left(E_{1}, E_{3}\right) \varphi E_{1}=0 .
$$

Similarly, we can verify other results. Hence we have

$$
\left(\nabla_{X} \varphi\right) Y=g(\varphi X, Y) \xi-\eta(Y) \varphi X \quad \text { for } \quad E_{3}=\xi .
$$

Thus, the first condition (2.4) for Kenmotsu manifold is also satisfied. Satisfying two conditions (2.4) and (2.5) for Kenmotsu manifold, the manifold under consideration is a 3-dimensional Kenmotsu manifold. By the definition of Riemannian curvature tensor in terms of $\nabla$, we have

$$
R(X, Y) Z=\nabla_{X} \nabla_{Y} Z-\nabla_{Y} \nabla_{X} Z-\nabla_{[X, Y]} Z
$$

Thus, using definition of $R$, we have

$$
\begin{aligned}
R\left(E_{1}, E_{2}\right) E_{3} & =\nabla_{E_{1}} \nabla_{E_{2}} E_{3}-\nabla_{E_{2}} \nabla_{E_{1}} E_{3}-\nabla_{\left[E_{1}, E_{2}\right]} E_{3} \\
& =\nabla_{E_{1}} E_{2}-\nabla_{E_{2}} E_{1} \\
& =0 .
\end{aligned}
$$


Similarly, we obtain

$$
\begin{array}{r}
R\left(E_{2}, E_{3}\right) E_{3}=-E_{2}, R\left(E_{1}, E_{3}\right) E_{3}=-E_{1}, R\left(E_{1}, E_{2}\right) E_{2}=-E_{1}, \\
R\left(E_{2}, E_{3}\right) E_{2}=E_{3}, R\left(E_{1}, E_{3}\right) E_{2}=0, R\left(E_{1}, E_{2}\right) E_{1}=E_{2}, \\
R\left(E_{2}, E_{3}\right) E_{1}=0, R\left(E_{1}, E_{3}\right) E_{1}=E_{3} .
\end{array}
$$

From above curvature relations, it follows that $\varphi^{2}\left(\left(\nabla_{W} R\right)(X, Y) Z\right)=0$. Hence 3-dimensional Kenmotsu manifold is locally $\varphi$-symmetric.

\section{References}

[1] Blair DE (1976) Contact manifolds in Riemannian geometry, Lecture Notes in Mathematics, 509, Springer-Verlag, Berlin.

[2] Blair DE (2002) Riemannian geometry of contact and sympletic manifolds, Progress in Mathematics, 203, Birkhauser Boston, Inc., Boston.

[3] De UC and Shaikha AA (2007) Differential geometry of manifolds, Narosa Publishing House, New Delhi.

[4] Hicks NJ (1965) Notes on differential geometry, D. Van Nostrand Co., Inc., Princeton, New York.

[5] Kenmotsu K (1972) A class of almost contact Riemannian manifold, Tohoku Math. J., 24, 93 - 103.

[6] Mishra RS (1984) Structures on a differentiable manifold and their applications, Chandrama Prakashan, Allahabad.

[7] Takahashi T (1977) Sasakian $\varphi$-symmetric spaces, Tohoku Math. J., 29, 91 - 113.

[8] Tanno S (1969) The automorphism groups of almost contact Riemannian manifolds, Tohoku Math. J., 21, $21-38$. 